\title{
Experimental realization of broadband control of water-wave-energy amplification in chirped arrays
}

\author{
A. J. Archer $\odot,{ }^{1,2}$ H. A. Wolgamot,,${ }^{1,2}$ J. Orszaghova $\odot,{ }^{1,2}$ L. G. Bennetts $\odot,^{3, *}$ \\ M. A. Peter ${ }^{4,5}$ and R. V. Craster ${ }^{6,7}$ \\ ${ }^{1}$ Faculty of Engineering, Computing and Mathematics, University of Western Australia, WA 6009, Australia \\ ${ }^{2}$ Wave Energy Research Centre, University of Western Australia, WA 6009, Australia \\ ${ }^{3}$ School of Mathematical Sciences, University of Adelaide, SA 5005, Australia \\ ${ }^{4}$ Institute of Mathematics, University of Augsburg, 86135 Augsburg, Germany \\ ${ }^{5}$ Augsburg Centre for Innovative Technologies, University of Augsburg, 86135 Augsburg, Germany \\ ${ }^{6}$ Department of Mathematics, Imperial College London, London SW7 2AZ, United Kingdom \\ ${ }^{7}$ Department of Mechanical Engineering, Imperial College London, London SW7 2AZ, United Kingdom
}

(Received 18 April 2020; accepted 28 May 2020; published 24 June 2020)

\begin{abstract}
Water waves in natural environments are typically broadband, nonlinear, and dynamic phenomena. Taking concepts developed for slow light in optics, we address the challenge of designing arrays to control the spatial distribution of wave energy, and amplify specific frequencies from within target frequency bands at individual locations. Meter-scale waveflume experiments, in which incident waves interact with a chirped array of eight vertical cylinders, demonstrate significant amplifications over a broad frequency range, as predicted numerically and theoretically.
\end{abstract}

DOI: 10.1103/PhysRevFluids.5.062801

\section{INTRODUCTION}

The ability of chirped (or graded) arrays to create slow light or sound waves, confine them, and filter them spatially according to frequency is attractive for wave control. This is often connected with rainbow trapping of light [1] or sound [2,3], and, when combined with the optical properties of surface plasmon polaritons [4], has led to the development of surface dispersion engineering devices based on chirped gratings [5] or tapered devices [6]. A recurring theme is that by using a graded surface grating, different wavelengths are trapped at different locations. Improvements in design and fabrication of nanopatterned metal surfaces, three-dimensional (3D) printing of surfaces, and optimization of their optical and acoustic properties open new applications to exploit the effect in light storage, energy harvesting, filtering, and delayed delivery for novel devices [7].

This comprehensive body of work, primarily in optics, plasmonics and electromagnetism, forms a paradigm for the control of waves more broadly, and, for example, has been extended to the field of elasticity [8-10], where it is used to generate mode-conversion devices that hybridize surface waves to body waves. A feature less often exploited is that energy can accumulate in regions where the group velocity tends to zero [11-13]. Assuming the local behavior in the array is dominated by neighboring elements enables the implied local periodicity to predict band gaps (or forbidden frequencies), and the band-gap edges determine a relation between local array properties and frequencies at which group velocities are zero. This interpretation empowers the design of broadband arrays with spatial filtering by frequency and localized amplification, and has recently been taken into linear water-wave theory using an array of fixed bottom-mounted cylinders [14].

*1uke.bennetts@adelaide.edu.au 
At present, lensing techniques are the standard approach for controlling the spatial distribution of water-wave energy, with a series of studies in the context of ocean-wave-energy harvesting beginning with pioneering work in the early 1980s, e.g., Ref. [15]. Lenses formed from arrays of hundreds of fixed bottom-mounted cylinders have been proposed, using superlensing concepts, created by negative refraction [16], biconvexivity [17,18], and gradient indexing [19]. Experimental realizations of the lenses have been made on the millimetric scale, with focal points created several to tens of wavelengths behind the lenses, in which amplitude amplifications of approximately factor two and weak dependence of the focal location on wave frequency have been reported. A conceptually different sort of water-wave lens formed by an annular structure of radial slits has also been proposed, using ideas from transformation optics, with experimental realization at the millimetric amplitude scale, achieving amplitude amplification up to factor three [20].

In this Rapid Communication, we address the pressing question of whether it is possible to take advantage of generic rainbow-trapping concepts in a (potentially nonlinear and dissipative) water-wave system, to yield large amplifications of chosen frequencies at well-separated locations. We do so through careful experimentation on waves of length-order meters, interacting with an array of eight identical fixed bottom-mounted cylinders of radius $0.25 \mathrm{~m}$ arranged along the centerline of a 1.49-m-wide, 54-m-long, 1.6-m-tall wave flume, and with chirped spacing, similar to Ref. [21] but with the cylinder spacing increasing in the incident wave direction (Fig. 1). The cylinders span the full height of the flume, which is filled with water to $1.1 \mathrm{~m}$ depth. A hinged wavemaker at one end of the flume generates incident waves, and those transmitted through the array are absorbed by a passive beach, which reflects $<1 \%$ of the energy reaching it (in the frequency range of interest).

\section{RESULTS}

\section{A. Band-gap theory and numerical simulations}

Analysis of doubly periodic infinite arrays - with periodicity in the propagation direction $(x$ direction) matching the local cylinder spacing, and the periodicity in the transverse direction matching the flume width (analogous to the walls) - yields valuable information to guide the experimental tests. The band diagram for the doubly-periodic array (i.e. dispersion curves; see Supplemental Material [23]) associated with the third spacing [Fig. 2(a)] shows well-defined band gaps, with the first two bands well separated from the more densely arranged bands above, and informs choices of incident frequencies. We choose to operate towards the upper end of the second band as the wavelength is smaller than on the first band, so the waves scatter more strongly from the cylinders, travel more slowly, and are in deep water relative to the wavelength. The second band is very flat close to the upper band edge over a large range of wave numbers, generating a high density of states, and enabling us to harness the "flat-band slow light," thereby reducing dispersion [22].

Computed steady-state linear free-surface responses around the array, driven by monochromatic incident waves, are shown in Figs. 2(c) and 2(d). The almost flat dispersion curve acts to slow the wave within the array, and the cylinder spacings at which the band gap causes propagation to cease are predicted [Fig. 2(b)]. The linear theory for an infinite array, implicitly assuming adiabatic grading of the array (based on the gradual grading of cylinder spacing, where adjacent spacings differ by $<10 \%$ ), is remarkably accurate in its predictions even for merely eight cylinders. Moreover, the amplitude amplifications in the cylinder spacings before propagation ceases are factors 9 and 17 for $\omega=6.50 \mathrm{rad} \mathrm{s}^{-1}$ [wavelength $1.46 \mathrm{~m}$; Fig. 2(d)] and $\omega=6.60 \mathrm{rad} \mathrm{s}^{-1}$ [wavelength $1.41 \mathrm{~m}$; Fig. 2(c)], respectively.

\section{B. Wave-flume experiments}

Given the predictions of such large amplifications at precise locations, the wave-flume experiments are used to explore the limitations of linear theory, and whether assumptions, such as ignoring viscosity, surface tension, and wave breaking, alter these predictions in practice. We probe the resonant modes using regular incident waves of angular frequencies $\omega=6.50$ and $6.60 \mathrm{rad} \mathrm{s}^{-1}$ 

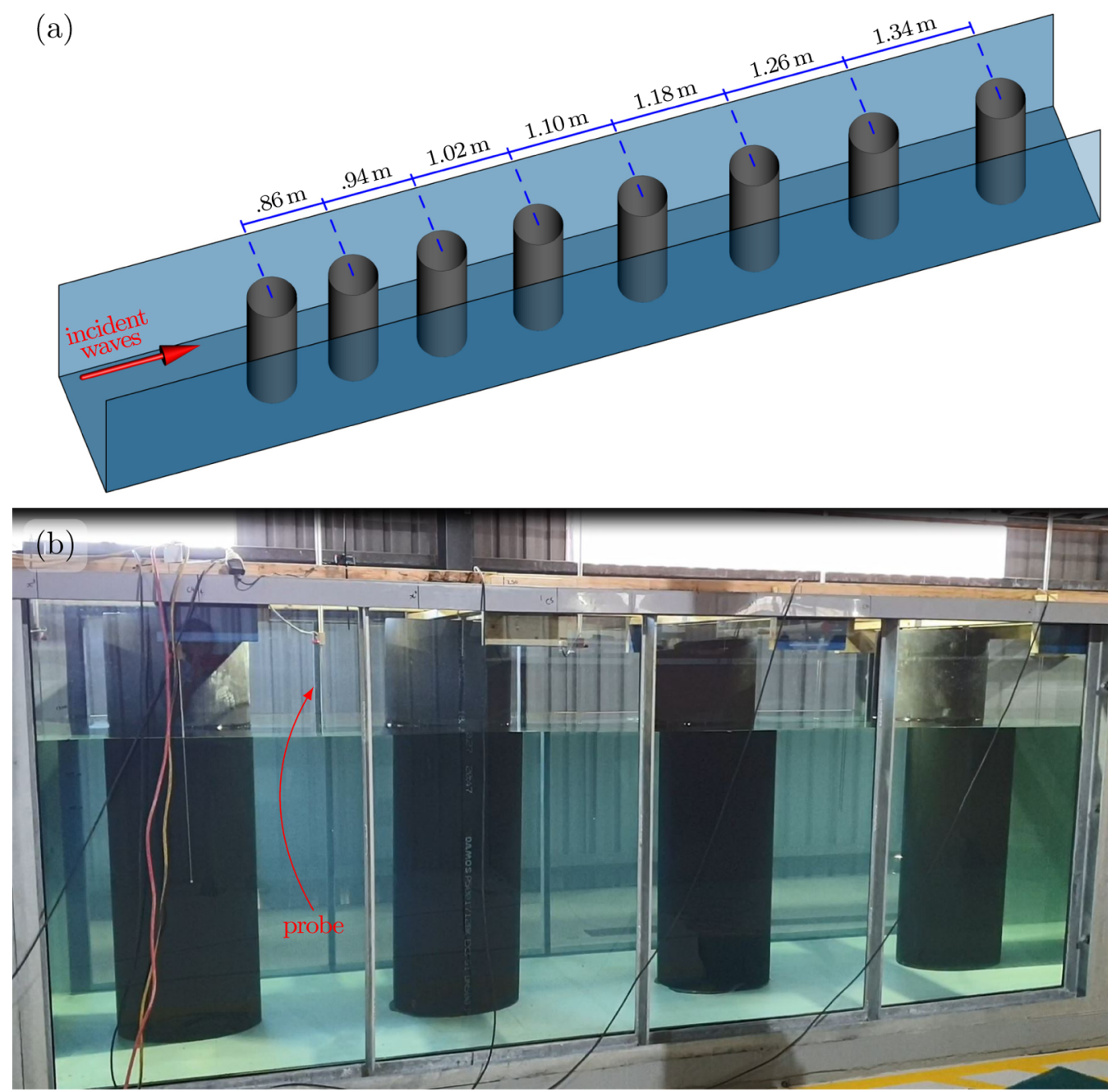

FIG. 1. (a) Wave-flume experimental setup, involving a chirped array of eight identical cylinders, with spacing increasing in the incident wave direction. (b) Side-view photograph showing cylinders 3-6, including probes in cylinder spacings.

[for comparison with theoretical responses in Figs. 2(c) and 2(d)], and amplitude $a_{\text {inc }}=0.01 \mathrm{~m}$. An incident wave of frequency $\omega=3.24 \mathrm{rad} \mathrm{s}^{-1}$ and amplitude $a_{\text {inc }}=0.03 \mathrm{~m}$, which exists on the first band and does not intersect a band edge, is also tested. Eulerian point measurements of the free-surface elevation are recorded at the center of each of the first six spacings (Fig. 2 suggests the maximum response will be approximately centered between cylinders). Linear time-series responses for $\omega=6.50$ and $6.60 \mathrm{rad} \mathrm{s}^{-1}$ [Figs. 3(d)-3(i)] show large amplifications in spacing 3 and spacing 2, respectively, and minimal transmission farther along the array, consistent with theoretical predictions. Further, similar mode shapes to those predicted by linear theory were observed, as indicated by the wetted surface of the cylinders [Fig. 3(j)]. In contrast, the incident wave at frequency $\omega=3.24 \mathrm{rad} \mathrm{s}^{-1}$ propagates through the array almost unchanged [Figs. 3(a)-3(c)]. 

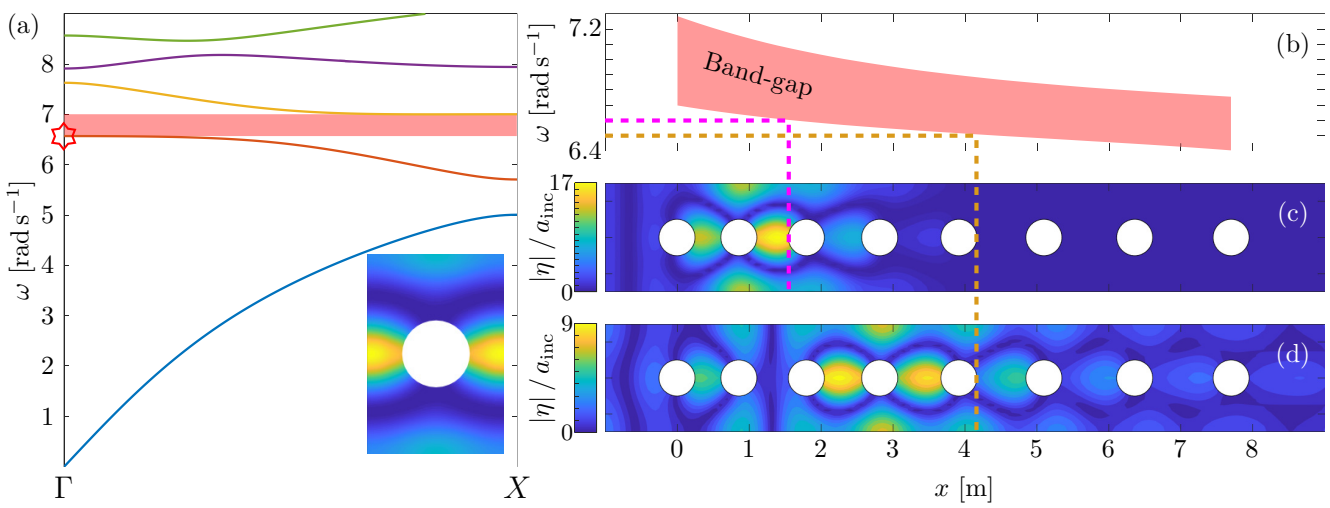

FIG. 2. (a) Band diagram (dispersion curves) of symmetric modes for infinite array, using third cylinder spacing for $x$ periodicity, where the abscissa covers wave-number space for normal incidence in the first irreducible Brillouin zone from in phase $(\Gamma)$ to out of phase $(X)$, and red shading indicates the second band gap. The inset shows the eigenmode on the second band at $\Gamma$, as indicated by the red star. (b) Red shading shows the evolution of the second band gap as the cylinder spacing increases along the array, where spacing is interpolated between spacing midpoints and extrapolated at the ends. (c), (d) Model predictions of free-surface-elevation modulus $|\eta|$, normalized by the incident amplitude $a_{\text {inc }}$, for regular incident waves with angular frequency (c) $\omega=6.60 \mathrm{rad} \mathrm{s}^{-1}$ and (d) $\omega=6.50 \mathrm{rad} \mathrm{s}^{-1}$. Dashed curves connecting (b) with (c) and (d) indicate theoretical predictions of zero group velocity, where $\omega=6.60 \mathrm{rad} \mathrm{s}^{-1}$ (upper dashed, magenta curve) and $\omega=6.50 \mathrm{rad} \mathrm{s}^{-1}$ (lower dashed, orange curve) enter the band gap.

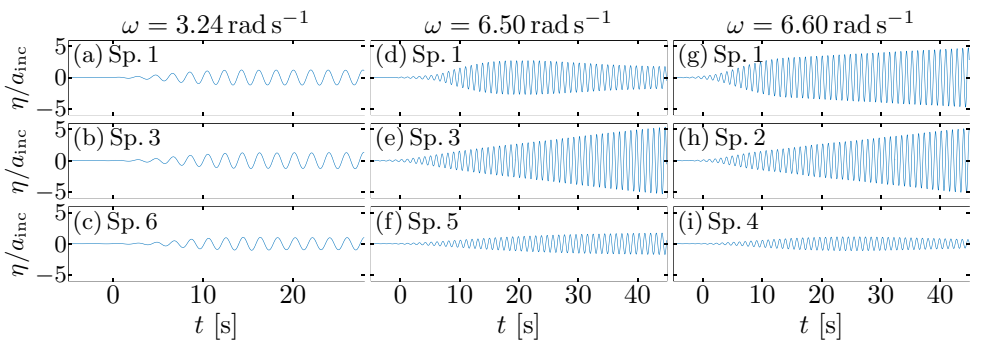

(k) Spacing 1

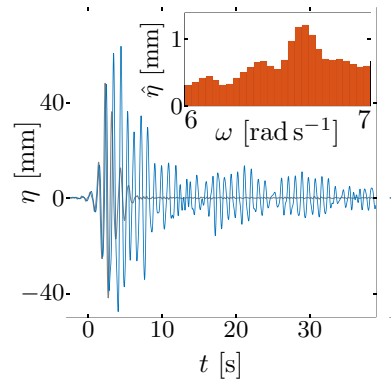

(1) Spacing 2

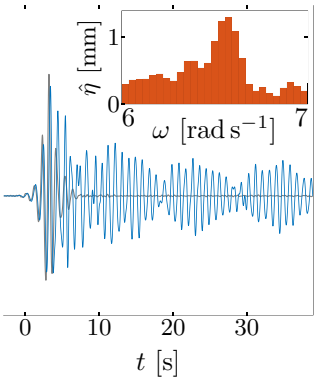

(m) Spacing 3

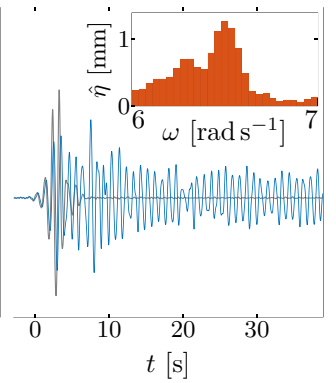

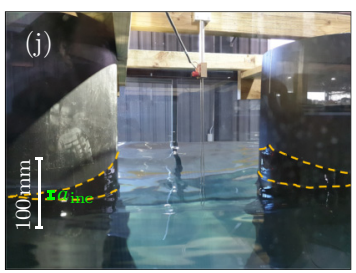

(n) Spacing 4

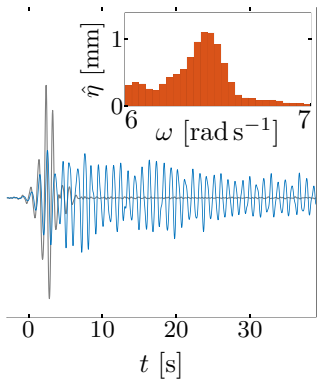

FIG. 3. (a)-(i) Linearized experimental time series for regular incident waves at frequency (a)-(c) $\omega=$ $3.24 \mathrm{rad} \mathrm{s}^{-1}$, (d)-(f) $\omega=6.50 \mathrm{rad} \mathrm{s}^{-1}$, and (g)-(i) $\omega=6.60 \mathrm{rad} \mathrm{s}^{-1}$, with responses shown in selected spacings, and where $t=0$ is the incident wave arrival time. (j) Photograph of resonant mode shape in spacing 3 for $\omega=6.50 \mathrm{rad} \mathrm{s}^{-1}$. (k)-(n) Experimental time series for focused incident wave packet in spacings 1-4 (solid blue curve), with corresponding incident packets superimposed (solid gray curve). The insets show spectral decompositions of responses. 

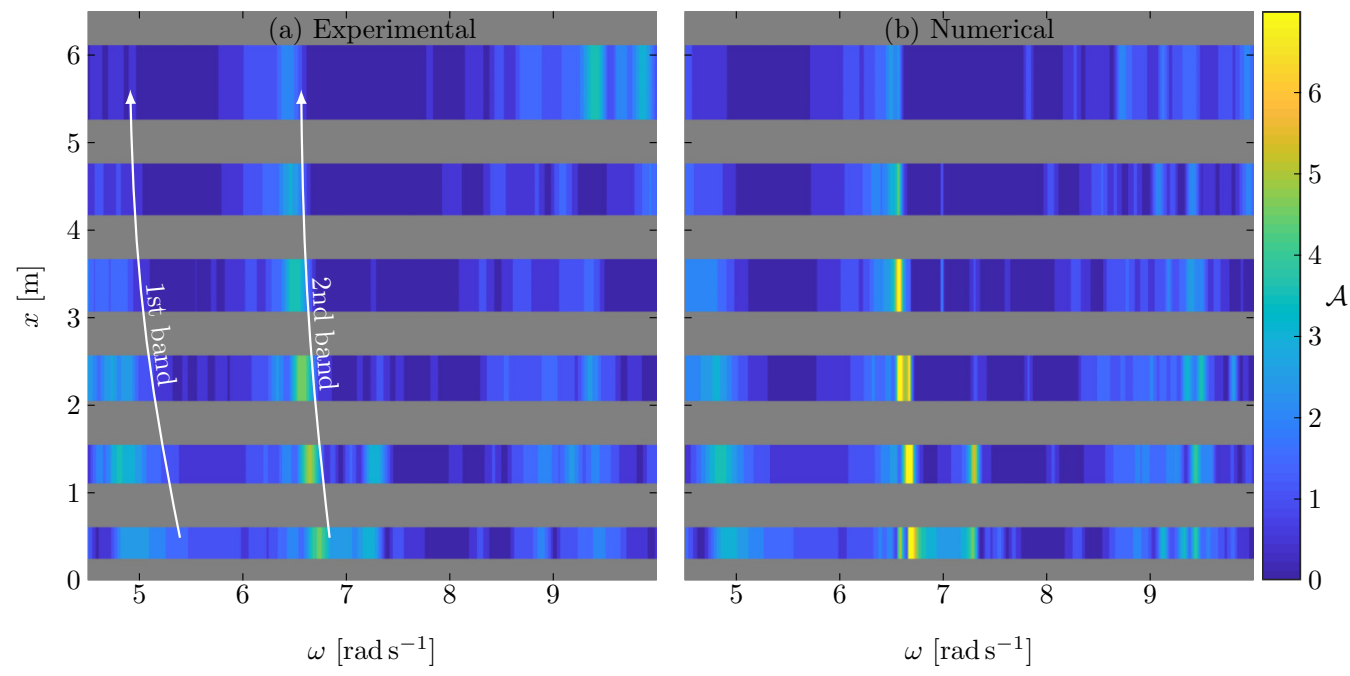

FIG. 4. (a) Experimental and (b) numerical amplitude transfer functions, at wave probe locations along the array (shown as constant in each spacing). Gray strips represent spatial locations of cylinders 1-7.

Focused incident wave packets are used to investigate the overall spectral structure of the response. The propagating linear free-surface signal created at the wavemaker is

$$
\eta_{\text {inc }}(\tau)=A_{\text {inc }} \frac{\sum_{n}\left\{S\left(\omega_{n}\right) \cos \left(k_{n} x_{0}-\omega_{n} \tau\right)\right\}}{\sum_{n} S\left(\omega_{n}\right)},
$$

where $A_{\text {inc }}=0.045 \mathrm{~m}$ is the nominal amplitude, $x_{0}=-24 \mathrm{~m}$ is the mean wavemaker position relative to the linear focal point, $\tau$ is time relative to linear focus time, $k_{n}$ is the wave number, and $S$ is a Gaussian with standard deviation $\sigma=0.15 \mathrm{rad} \mathrm{s}^{-1}$ and mean $\omega_{\mathrm{p}}=6.03 \mathrm{rad} \mathrm{s}^{-1}$, i.e., a frequency on the second band. The linear focus position is $2 \mathrm{~m}$ into the array and the sum is over $2^{10}$ equally spaced components. Figures 3(k)-3(n) show raw surface-elevation time-series responses in spacings $1-4$, with the corresponding incident series superimposed for comparison (from tests without cylinders). The insets show the response amplitude spectra in $0.04 \mathrm{rad} \mathrm{s}^{-1}$ frequency bins, from the (discrete) Fourier transform of the surface-elevation time series, $\hat{\eta}=|\mathcal{F}(\eta)|$. The spectral peak occurs on the second band, and is frequency downshifted from $6.66 \pm 0.02$ to $6.42 \pm 0.02 \mathrm{rad} \mathrm{s}^{-1}$ over spacings 1-4, consistent with the theoretical predictions (cf. Fig. 2). Moreover, the small responses for frequencies above the peaks in spacings 2-4 are consistent with the theoretical predictions of the second band gap [Fig. 2(b)].

\section{Broadband response: Experimental and numerical agreement}

A transfer function is defined as

$$
\mathcal{A}=\frac{\hat{\eta}}{\hat{\eta}_{\text {inc }}} \quad \text { where } \quad \hat{\eta}_{\text {inc }}=\left|\mathcal{F}\left(\eta_{\text {inc }}\right)\right|,
$$

to quantify amplitude amplifications over the frequency spectrum. Figure 4 shows the amplitude transfer function calculated from the numerical model (for which $\mathcal{A}=|\eta| / a_{\text {inc }}$ ) and from focused wave group experiments [averaged over the test shown in Figs. 3(k)-3(n) and tests with a shifted peak frequency $\omega_{\mathrm{p}}=6.55 \mathrm{rad} \mathrm{s}^{-1}$ and shifted amplitude $\left.A_{\text {inc }}=0.029 \mathrm{~m}\right]$. The experimental and numerical transfer functions are in excellent agreement, notwithstanding the expected larger and sharper amplifications in the numerical model. The amplifications on the second band are evident, 


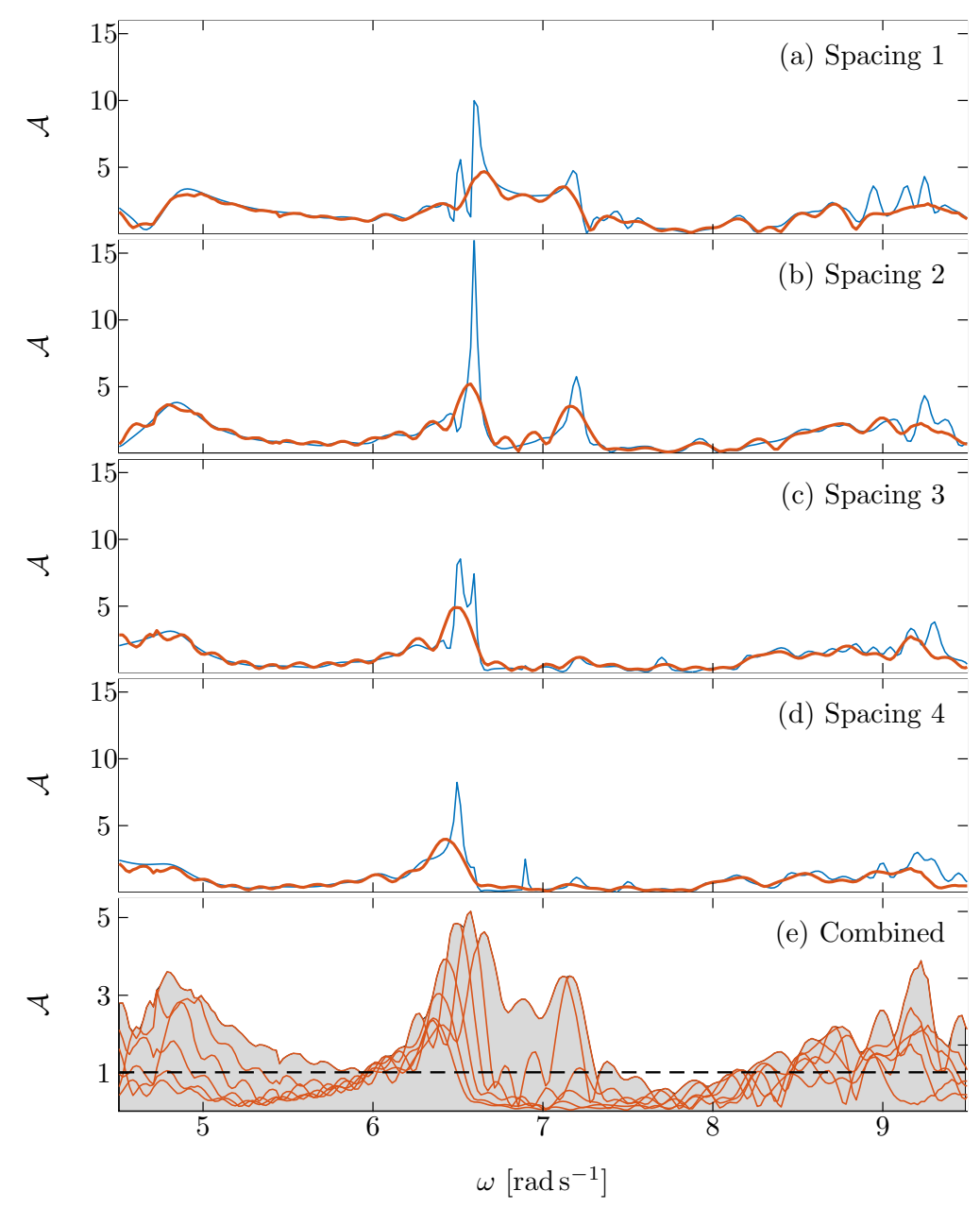

FIG. 5. (a)-(d) Experimental (solid orange curve) and numerical (solid blue curve) amplitude transfer functions in spacings 1-4. (e) Combined experimental transfer functions for spacings 1-6 (solid orange curve) with envelope (gray shaded region), and amplification boundary (dashed black curve).

along with the spectral downshift along the array. Cognate amplifications are also shown for the first band (see Supplemental Material [23] Fig. S3).

Figures 5(a)-5(d) emphasize the numerical-experimental agreement for spacings 1-4. The broadband amplification given by the array is illustrated in Fig. 5(e), which shows the experimental transfer functions for spacings $1-6$, and with the envelope indicated by the gray shading. The majority of the envelope lies well above the boundary $\mathcal{A}=1$, which divides amplification $(\mathcal{A}>1)$ from suppression $(\mathcal{A}<1)$, with the only interval experiencing suppression from 7.45 to $8.10 \mathrm{rad} \mathrm{s}^{-1}$. The maximum amplifications are $\approx 5$, and occur on the second band, with corresponding energy amplifications $>20$. Amplifications $>3$ also occur over multiple frequency intervals, giving energy amplifications $\approx 10$.

\section{Resonance timescales}

In Figs. 5(a)-5(d), the numerical and experimental transfer functions overlap, except at the most resonant peaks, where the numerical predictions are larger and sharper than the experimental 
realizations. The discrepancies at the resonances are expected, as the numerical model neglects dissipative and nonlinear phenomena. However, the discrepancies are also attributed to difficulties in capturing the full resonance timescales during the experiments, as the time series (Fig. 3) are truncated just prior to the point when waves reflected by the array, then rereflected by the wavemaker, return to contaminate the results. This results in the experimental data underestimating the maximum amplifications.

In the regular wave tests [Figs. 3(a)-3(i)], the resonances have timescales longer than the test window available (Supplemental Material [23] Fig. S1) so amplitudes are still increasing towards a steady state at the end of the window, and the maximum possible amplifications are not achieved [Figs. 3(e), 3(g) and 3(h)]. Numerical simulations do not suffer from contamination by spurious reflections, and suggest substantial increases in amplitude from 50 to $100 \mathrm{~s}(35 \%$ for $\omega=6.50 \mathrm{rad} \mathrm{s}^{-1}$ and $55 \%$ for $\omega=6.60 \mathrm{rad} \mathrm{s}^{-1}$ ), and comparable increases from then until the steady states are reached for $t>300 \mathrm{~s}$ (Supplemental Material [23] Fig. S2), although it is likely that viscous dissipation would result in smaller increases in the experiments if the test windows could be elongated. Similarly, in the focused packet tests [Figs. 3(k)-3(n)], truncation of the time series cuts off the slow resonant decays, so that the maximum amplifications are not captured in the mapping to frequency space (i.e., by the Fourier transform).

\section{SUMMARY}

We have presented results from wave-flume experiments that demonstrate localization and amplification of water waves along a chirped array of bottom-mounted cylinders, despite the challenges of working with highly resonant systems in a finite-length flume. Linear theory was shown to predict the amplification spectrum accurately in frequency-distance space, including peak resonance locations and spectral downshifts along the array. Moreover, even for the rather small array of eight cylinders used, the amplification locations, at which the group velocity slows to zero, were shown to be consistent with the band structure of infinite periodic arrays using local cylinder spacings. The outcomes suggest future strategies for ocean-wave-energy harvesting by arrays should consider taking advantage of the broadband response and precise control demonstrated here. We have not attempted a parametric study to optimize such an array; this will be a future undertaking.

\section{ACKNOWLEDGMENTS}

We thank the anonymous reviewer for his/her comments. A.J.A. was supported by the Wave Energy Research Centre, jointly funded by The University of Western Australia and the Western Australian Government, via the Department of Primary Industries and Regional Development (DPIRD). H.A.W. acknowledges financial support from Shell Australia. L.G.B. is supported by an Australian Research Council midcareer fellowship (FT190100404). R.V.C. thanks the U.K. EPSRC for their support through Programme Grant No. EP/L024926/1 and also acknowledges the support of the Leverhulme Trust.

[1] K. L. Tsakmakidis, A. D. Boardman, and O. Hess, Trapped rainbow storage of light in metamaterials, Nature (London) 450, 397 (2007).

[2] J. Zhu, Y. Chen, X. Zhu, F. J. Garcia-Vidal, X. Yin, W. Zhang, and X. Zhang, Acoustic rainbow trapping, Sci. Rep. 3, 1728 (2013).

[3] N. Jimenez, V. Romero-Garcia, V. Pagneux, and J.-P. Groby, Rainbow-trapping absorbers: Broadband, perfect and asymmetric sound absorption by subwavelength panels for transmission problems, Sci. Rep. 7, 13595 (2017).

[4] J. B. Pendry, L. Martin-Moreno, and F. Garcia-Vidal, Mimicking surface plasmons with structured surfaces, Science 305, 847 (2004). 
[5] Q. Gan and F. J. Bartoli, Surface dispersion engineering of planar plasmonic chirped grating for complete visible rainbow trapping, Appl. Phys. Lett. 98, 251103 (2011).

[6] C. Argyropoulos, K. Q. Le, N. Mattiucci, G. D’Aguanno, and A. Alù, Broadband absorbers and selective emitters based on plasmonic brewster metasurfaces, Phys. Rev. B 87, 205112 (2013).

[7] S. V. Boriskina, H. Ghasemi, and G. Chen, Plasmonic materials for energy: From physics to applications, Mater. Today 16, 375 (2013).

[8] D. Cardella, P. Celli, and S. Gonella, Manipulating waves by distilling frequencies: A tunable shuntenabled rainbow trap, Smart Mater. Struct. 25, 085017 (2016).

[9] S. Krodel, N. Thome, and C. Daraio, Wide band-gap seismic metastructures, Extreme Mech. Lett. 4, 111 (2015).

[10] A. Colombi, D. Colquitt, P. Roux, S. Guenneau, and R. V. Craster, A seismic metamaterial: The resonant metawedge, Sci. Rep. 6, 27717 (2016).

[11] V. Romero-Garcia, R. Pico, A. Cebrecos, V. J. Sanchez-Morcillo, and K. Staliunas, Enhancement of sound in chirped sonic crystals, Appl. Phys. Lett. 102, 091906 (2013).

[12] Y. Chen, H. Liu, M. Reilly, H. Bae, and M. Yu, Enhanced acoustic sensing through wave compression and pressure amplification in anisotropic metamaterials, Nat. Commun. 5, 5247 (2014).

[13] L. G. Bennetts, M. A. Peter, and R. V. Craster, Low-frequency wave-energy amplification in graded two-dimensional resonator arrays, Philos. Trans. R. Soc., A 377, 20190104 (2019).

[14] L. G. Bennetts, M. A. Peter, and R. V. Craster, Graded resonator arrays for spatial frequency separation and amplification of water waves, J. Fluid Mech. 854, R4 (2018).

[15] J. J. Stamnes, O. Løvhaugen, B. Spjelkavik, C. C. Mei, E. Lo, and D. K. P. Yue, Nonlinear focusing of surface waves by a lens-theory and experiment, J. Fluid Mech. 135, 71 (1983).

[16] X. Hu, Y. Shen, X. Liu, R. Fu, and J. Zi, Superlensing effect in liquid surface waves, Phys. Rev. E 69, 030201(R) (2004).

[17] X. Hu and C. T. Chan, Refraction of Water Waves by Periodic Cylinder Arrays, Phys. Rev. Lett. 95, 154501 (2005).

[18] J. Yang, Y. F. Tang, C. F. Ouyang, X. H. Liu, X. H. Hu, and J. Zi, Observation of the focusing of liquid surface waves, Appl. Phys. Lett. 95, 094106 (2009).

[19] Z. Wang, P. Zhang, X. Nie, and Y. Zhang, Focusing of liquid surface waves by gradient index lens, Europhys. Lett. 108, 24003 (2014).

[20] C. Li, L. Xu, L. Zhu, S. Zou, Q. H. Liu, Z. Wang, and H. Chen, Concentrators for Water Waves, Phys. Rev. Lett. 121, 104501 (2018).

[21] A. Cebrecos, R. Picó, V. J. Sánchez-Morcillo, K. Staliunas, V. Romero-García, and L. M. Garcia-Raffi, Enhancement of sound by soft reflections in exponentially chirped crystals, AIP Adv. 4, 124402 (2014).

[22] J. Li, T. P. White, L. O'Faolain, A. Gomez-Iglesias, and T. F. Krauss, Systematic design of flat band slow light photonic crystal waveguides, Opt. Express 16, 6227 (2008).

[23] See Supplemental Material at http://link.aps.org/supplemental/10.1103/PhysRevFluids.5.062801 for information on the band diagrams, growth of resonant responses, amplifications on the first band, and experimental repeatability, which includes Refs. [24,25,26].

[24] R. Eatock Taylor, On modelling the diffraction of water waves, Ship Technol. Res. 54, 54 (2007).

[25] S. W. Joo, W. W. Schultz, and A. F. Messiter, An analysis of the initial-value wavemaker problem, J. Fluid Mech. 214, 161 (1990).

[26] P. McIver, Water-wave propagation through an infinite array of cylindrical structures, J. Fluid Mech. 424, 101 (2000). 